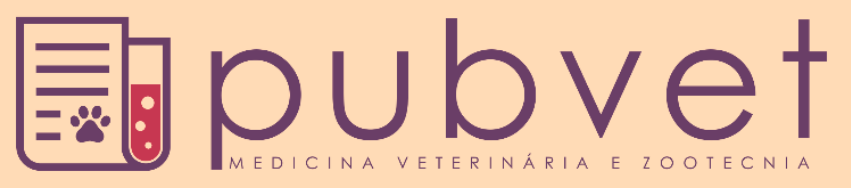

https://doi.org/10.31533/pubvet.v15n11a952.1-14

\title{
Tríade canina na Leishmaniose Visceral: Reservatório-vítima-sentinela
}

\author{
Adam Leal-Lima $^{1^{*} \bullet}$, Társsila Mara Vieira Ferreira ${ }^{2}$, Luana Oliveira Leite $^{2} \theta$, Bruna Leal \\ ${\text { Lima } \text { Maciel }^{30} \mathbb{0} \text {, Diana Celia Sousa Nunes-Pinheiro }}^{30}$
}

${ }^{I}$ Doutorando do Programa de Pós-Graduação em Ciências Veterinárias, Universidade Estadual do Ceará, Fortaleza/CE, Brasil ${ }^{2}$ Doutoranda da Programa de Pós-Graduação em Ciências Veterinárias, Universidade Estadual do Ceará, Fortaleza/CE, Brasil. ${ }^{3}$ Professora do Programa de Pós-Graduação em Ciências Veterinárias, Universidade Estadual do Ceará, Fortaleza/CE, Brasil.

*Autor para correspondência, E-mail: adamlima86@hotmail.com

Resumo. O objetivo desta revisão é esclarecer o triplo papel, reservatório-vítima-sentinela, que o cão exerce na leishmaniose visceral e discutir o foco na eliminação do "melhor amigo do homem" para proteção do ecossistema, levando-se em conta os três pilares epidemiológicos, parasito-vetor-hospedeiro, e os aspectos éticos. Logo, inicialmente serão abordados aspectos epidemiológicos relacionados a Leishmaniose Visceral (LV) e a Leishmania Visceral Canina (LVC). Posteriormente, uma reflexão sobre o papel do cão neste contexto, tido como principal reservatório da Leishmania infantum no ambiente urbano, será realizada visando a compreensão de medidas como a eutanásia, de animais positivos; a manutenção do cão parasitado na família (vítima) e o uso destes animais como sentinelas para a detecção da LV e LVC. Finalmente, conclui-se que a LVC é um problema que aflige a família multiespécie brasileira, afetando seres humanos e cães. Os métodos de controle e vigilância paras a LV implementadas no Brasil ainda são ineficientes e desatualizados, desconsiderando o cão como membro da família e tratando-o unicamente como hospedeiro reservatório. Portanto é fundamental uma revisão no Programa de Controle e Vigilância que inclua a Saúde Única na forma de lidar com as leishmanioses: hospedeiros, vetores e parasitos, dentro de um mesmo ambiente para dirigir recursos em medidas eficientes, com o cão sendo utilizado como sentinela da LV.

Palavras-chave: Ética, monitoramento epidemiológico, saúde única, vigilância sanitária, zoonoses

\section{Canine victim-sentinel-reservoir triad in Visceral Leishmaniasis Dogs triple role in leishmaniosis}

Abstract. The aim of this review is to reinforce the canine reservoir-victim-sentinel triad in the LV and to argue that the LV Prevention and Control Program should change the focus on eliminating the "best friend of man" to protect the ecosystem, taking into account the three epidemiological pillars, parasite-vector-host, and ethical aspects. Therefore, epidemiological aspects related to Visceral Leishmaniasis (LV) and Canine Visceral Leishmania (LVC) were initially addressed. Subsequently, a reflection on the role of the dog in this context, considered as the main reservoir of Leishmania infantum in the urban environment, was carried out aiming at understanding measures such as euthanasia, in the case of positive animals; the maintenance of the sick dog in the family (victim of the parasite) and the use of these animals as sentinels for the detection of LV and LVC. Finally, it is concluded that LVC is a problem that afflicts the Brazilian multispecies family, affecting humans and dogs. The methods of control and surveillance for VL implemented in Brazil are still inefficient and outdated, disregarding the dog as a member of the family and treating it only as a reservoir host. Therefore, a reform in the Control and Surveillance Program that includes One Health in the way of dealing with leishmaniasis: hosts, vectors 
and parasites, within the same environment, is essential to direct resources in efficient measures, with the dog being used as sentinel of the LV.

Keywords: Epidemiological monitoring, ethics, health surveillance, one health, zoonoses

\section{Introdução}

A (LV) é a forma mais grave das leishmanioses com elevada mortalidade quando não tratada e letalidade geral em torno de 8\% (Oliveira \& Fernandes, 2014; OPAS, 2018). São contabilizados 50.000 casos de LV no mundo anualmente e países de alta endemicidade concentram 93\% dos casos, sendo Brasil um desses 10 países (WHO, 2017). Em ambientes urbanos, os cães são capazes de manter Leishmanina infantum circulante em um determinado espaço e tempo comportando-se como hospedeiros reservatórios da LV (Alencar, 1959; Carvalho et al., 2018).

No Brasil foi instituído o Decreto $\mathrm{N}^{\circ} 51.838$ de 14 de março de 1963 visando o combate as Leishmanioses, preconizando o monitoramento de cães em áreas endêmicas e aqueles positivos para LVC são eutanasiados. Isso promoveu uma dicotomia, reservatório-vítima, entre sanitaristas e as famílias, já que é considerada cruel e socialmente inaceitável por parte da sociedade brasileira (Costa, 2011; Dantas-Torres et al., 2018a). Contudo, não repercutiu na redução do número de casos, nem humanos e tampouco caninos (Courtenay et al., 2002; Nunes et al., 2010; Sousa-Paula et al., 2019).

Um cão infectado por $L$. infantum pode viver por anos quando monitorado por um médico veterinário e um tutor responsável (Andrade et al., 2011; Yasur-Landau et al., 2016). No entanto, é importante considerar os aspectos éticos, morais e legais do impacto da escolha na saúde humana, animal e ambiental (Irwin, 2014; Passantino et al., 2010). Portanto, controlar, monitorar, prevenir, tratar e curar LVC devem ser metas e estratégias dos programas de saúde.

Neste sentido, o conceito One Health propõe a promoção de uma abordagem interdisciplinar e multisetorial na promoção de uma saúde única integrada e continuada entre meio ambiente, seres humanos e animais (Evans \& Leighton, 2014; Zinsstag et al., 2012). Considerando que os casos caninos precedem casos humanos em incidência, prevalência (Fraga et al., 2012; Freitas et al., 2013; Lima et al., 2017; Lima et al., 2018; Palatnik-de-Sousa et al., 2009; Prado et al., 2011), epidemias (Mahmud et al., $\underline{2019}$ ) e expansão geográfica de endemias (Abrantes et al., 2018), sugere-se que a espécie canina seja utilizada como sentinela. Portanto, o objetivo desta revisão é discutir a condição de reservatório-vítimasentinela do cão na LV e assim reavaliar a centralização das ações de saúde pública brasileira na eliminação dos cães como reservatórios urbanos.

\section{Aspectos epidemiológicos da leishmaniose visceral no Brasil}

O Brasil possui uma epidemiologia singular de LV determinada pela espécie do protozoário endêmico $L$. infantum e pelo clima tropical que favorece a abundância do vetor biológico do gênero Lutzomyia (Afonso et al., 2005; Santos et al., 1998). Ressalte-se que fêmeas Lu. longipalpis são as mais abundantes (Almeida et al., 2010; Góes et al., 2012).

Protozoários do gênero Leishmania compreendem mais de 50 espécies identificadas pelo mundo (Figura 1) (Akhoundi et al., 2016). São parasitos dimórficos apresentando-se na forma amastigota, dentro de células do sistema fagocitário (Oikonomidis et al., 2019) dos mamíferos, e na forma promastigota flagelada, no sistema gastrointestinal dos vetores flebotomíneos (Séguin \& Descoteaux, $\underline{2016)}$.

Os agentes etiológicos das LV são a $L$. infantum, com ciclo biológico zoonótico (Figura 2) e a $L$. donovani, com ciclo biológico antroponótico (WHO, 2010). No Brasil, foi relatado que o principal agente etiológico causador de LVC é L. chagasi (Alencar, 1959), considerada sinonímia de L. infantum (Dantas-Torres, 2006).

A tabela 1 reúne dados de frequência para LVC no Brasil. Verifica-se uma variação na percentagem de cães infectados, podendo chegar até 50\% (Leça Júnior et al., 2015), o que demonstra a capacidade dos cães de manterem o agente infeccioso circulante. Além disso, a variação de casos caninos e humanos está relacionada a ciclicidade da carga de Leishmania spp. no espaço e tempo (Courtenay et al., 2017). Deve-se ressaltar que fatores ambientais, como temperatura, precipitação e avanço dos ambientes 
urbanos em áreas que eram cobertas de mata, podem interferir nos números de soropositividade de cães (Abrantes et al., 2018).

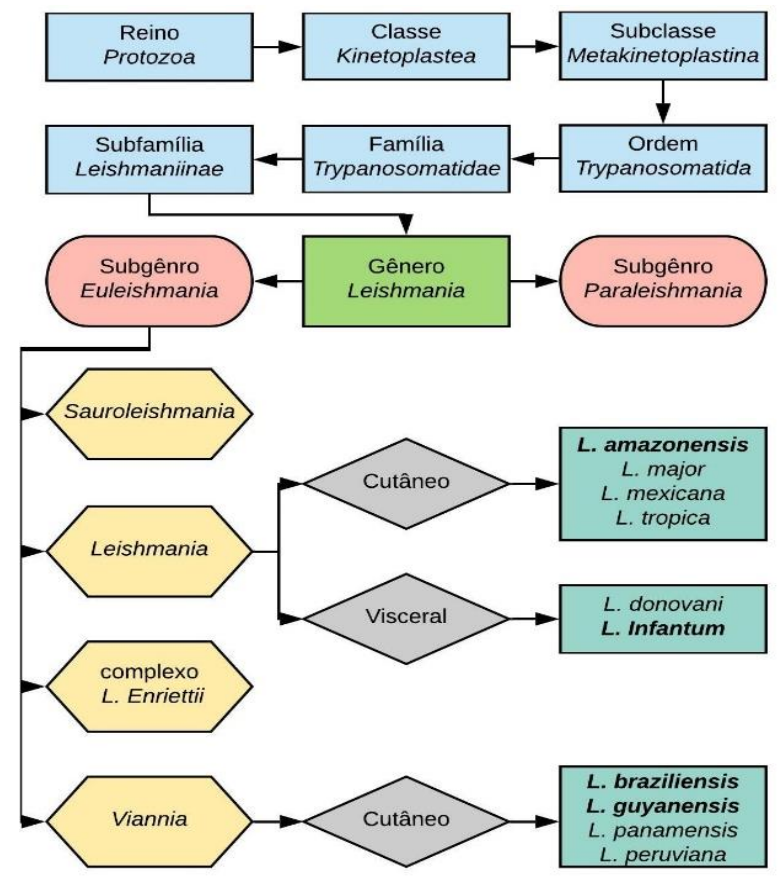

Figura 1. Espécies com importância patogênica no Brasil estão em negrito.

Cães, além de manterem a $L$. infantum no ambiente ao longo dos anos (Tabela 1), mantém a enzootia devido à transmissão por vias sexual e transplacentária (Figura 2). Machos infectados transmitem o parasito pelo coito (Silva et al., 2009), enquanto fêmeas infectadas transmitem aos seus descendentes, sendo essas vias suficientes para manter a expansão do ciclo enzoótico (Boggiatto et al., 2011; Toepp et al., 2019). Contudo, essas formas de transmissão não foram contempladas nos programas de vigilância e controle da LV no Brasil, o que implica na redução da eficiência de medidas profiláticas direcionadas ao vetor.

Tabela 1. Dados epidemiológicos da Leishmaniose Visceral Canina no Brasil.

\begin{tabular}{|c|c|c|c|c|c|}
\hline Autores, ano de publicação & Cães positivos & Amostra (n) & Diagnóstico & Estado & Ano de coleta \\
\hline $\begin{array}{l}\text { Dantas-Torres (2006) } \\
\text { (200) }\end{array}$ & $40,30 \%$ & 322 & Sorologia & $\mathrm{PE}$ & 2005 \\
\hline Figueiredo et al. (2018) & $6,90 \%$ & 1446 & Sorologia & SP, DF, TO, CE & 2008 \\
\hline Leça Júnior et al. (2015) & 50,30 ou $0,00 \%$ & 292 & Sorologia ou PCR & BA & 2010 \\
\hline Brito et al. (2016) & $8,40 \%$ & 391 & Sorologia & PB & 2010 \\
\hline Gonçalves. (2014) & $21,80 \%$ & 800 & Sorologia ou Cultura & BA & 2011 \\
\hline Fraga et al. (2016) & $14,10 \%$ & 780 & Análise de Classe Latente & BA & 2011 \\
\hline Abrantes et al. (2018) & $21,60 \%$ & 110 & Sorologia e/ou Cultura & RJ & 2011 \\
\hline Silva et al. (2016)) & $11,33 \%$ & 362 & Sorologia & PB & 2012 \\
\hline Ursine et al. (2016) & $19,50 \%$ & 487 & Sorologia & MG & 2013 \\
\hline Belo et al. (2017) & $8,90 \%$ & 1130 & Sorologia & MG & 2014 \\
\hline Leal et al. (2018) & $22,00 \%$ & 5822 & Sorologia & MG & 2014 \\
\hline Kazimoto et al. (2018) & $6,78-9,94 \%$ & 4727 & Sorologia & $\mathrm{RN}$ & 2014 \\
\hline Ribeiro et al. (2019) & $55,00 \%$ & 240 & Sorologia & DF & 2015 \\
\hline Dantas-Torres, et al. (2018b) & $31,60 \%$ & 95 & Sorologia & PE & 2015 \\
\hline Lana et al. (2018) & $14,80 \%$ & 9136 & Sorologia & MG & 2015 \\
\hline Carvalho et al. (2018) & $19,20 \%$ & 600 & Sorologia & MT & 2016 \\
\hline Fraga et al. $(\underline{2012)}$ & $1,00-17,00 \%$ & $529-16558$ & Sorologia & BA & $1997-2010$ \\
\hline Rondon et al. (2008) & $12,60-37,6 \%$ & 1381 & Sorologia & $\mathrm{CE}$ & $2005-2007$ \\
\hline Freitas et al. (2013) & $1,26-7,13 \%$ & $14761-126307$ & Sorologia & $\mathrm{CE}$ & $2006-2012$ \\
\hline Rocha et al. $(\underline{2018)}$ & $9,90 \%$ & 42112 & Sorologia & $\mathrm{AL}$ & $2007-2013$ \\
\hline Coura-Vital et al. (2014) & $6,20-8,10 \%$ & 1226 & Sorologia & MG & $2008-2010$ \\
\hline Sousa-Paula et al. (2019) & $0,50-8,10 \%$ & 13980 & Sorologia & $\mathrm{CE}$ & $2008-2017$ \\
\hline Rodrigues et al. (2017) & $3,53-8,90 \%$ & $84960-126307$ & Sorologia & $\mathrm{CE}$ & $2009-2013$ \\
\hline
\end{tabular}


No Brasil é crescente a resistência da população a eutanásia dos cães soropositivos (Costa, 2011; Zuben \& Donalísio, 2016), especialmente com a possibilidade do tratamento da LVC a partir de 2016 (Nota Técnica Conjunta ${ }^{\circ}$ 001/2016 MAPA/MS), bem como a propagação da vacina anti-Leishmania. Por outro lado, medicamentos utilizados para o tratamento da LVC são limitados em eficácia e possuem efeitos colaterais que acarretam sérios danos teciduais (Bekhit et al., 2018).

No Brasil, o tratamento do cão não é recomendado, pois não existem medidas que garantam a nãoinfectividade do cão em terapia (Portaria Interministerial No 1426 de 2008). Além disso, existe uma preocupação com a resistência às drogas leishmanicidas utilizadas no tratamento humano que limita o uso dessas drogas no tratamento canino. Cães tratados permanecem infectados por um longo período, pois os medicamentos utilizados apenas prolongam a vida do animal e melhoram sua condição clínica, com altas chances de recorrência dos sinais clínicos (Andrade et al., 2011; Manna et al., 2008; Woerly et al., 2009; Yasur-Landau et al., 2016).

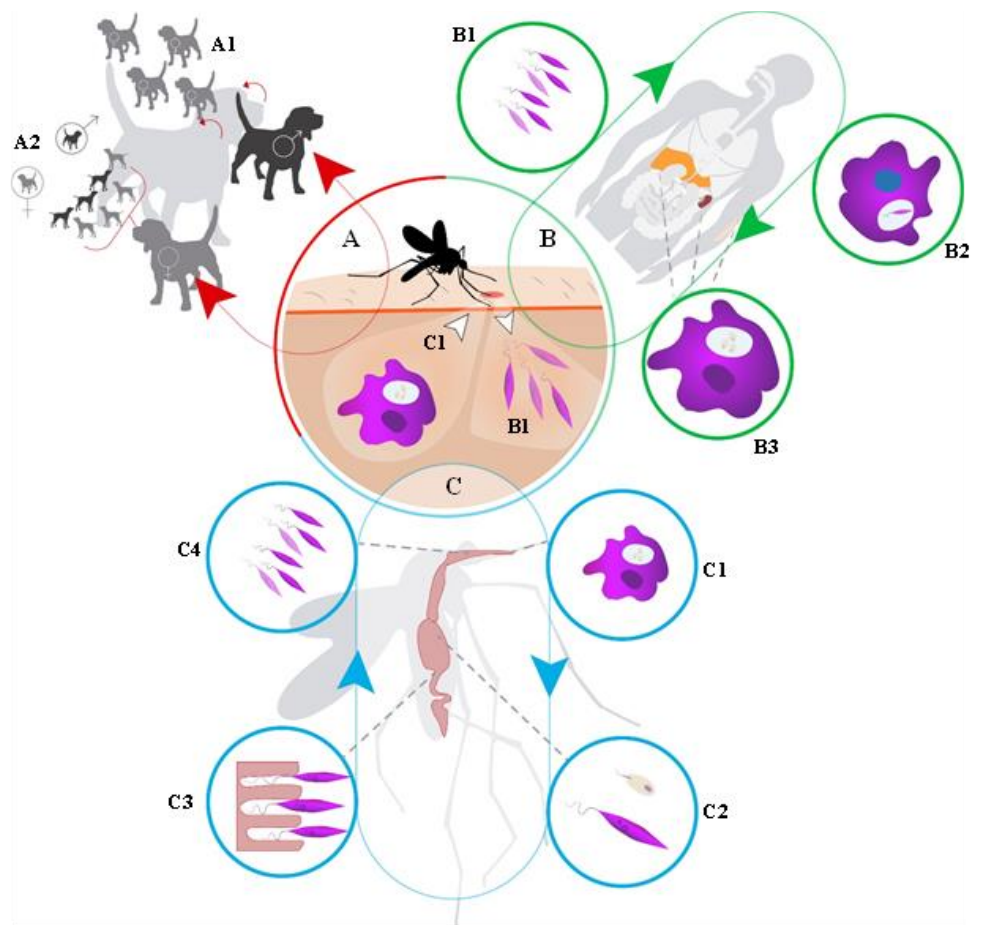

Figura 2. Vias de transmissão do parasito Leishmania infanutm. O vetor flebotomínio Lu. longipalpis adquire a forma amastigota de $L$. infantum dentro de fagócitos em mafíferos infectados $(\mathbf{C 1})$ e transmite a forma promastigota durante o repasto sanguíneo (B1). Sangue ingerido pelo flebotomíneo é digerido e o parasito muda de amastigota para promastigota (C2), as formas promastigotas amadurecem e se replicam no intestino do inseto $(\mathbf{C 3})$, tornando-se infectantes e migrando para a região anterior bucal do inseto (C4). L. infantum muda de forma dentro das células fagocitárias dos mamíferos, de promastigota para amastigotas (B2), e infecta novos órgãos, como médula óssea, fígado, baço e pele (B3). Cães infectados podem transmitir $L$ infantum por via sexual, machos para fêmeas (A1), e transplacentária (A2), mantendo o parasito no ambiente independente da presença do vetor.

\section{Cães como hospedeiro reservatório da Leishmania infantum}

Hospedeiros são animais que proveem comida e abrigo aos parasitos na relação de parasitismo e são classificados de diversas formas. Espécies do gênero Leishmania já foram identificadas em diversos mamíferos, destacando-se primatas, roedores, carnívoros, marsupiais, felinos (Pennisi \& Persichetti, 2018) e morcegos (Rezende et al., 2017). Por sua vez, hospedeiros reservatórios são animais/humanos capazes de manter o agente infeccioso no espaço/tempo determinado. Qualquer mamífero pode ser hospedeiro potencial desses parasitos, contudo poucos trabalhos evidenciam a capacidade das espécies infectadas de servir como hospedeiro reservatório (Roque \& Jansen, 2014).

O Programa de Controle das Leishmanioses do Ministério da Saúde do Brasil define reservatórios como mamíferos não humanos que infectam os vetores por longos períodos de tempo, e destaca a espécie Canis familiaris como reservatório urbano (Alencar, 1959; Brasil, 2014; Brasil, 2016; Otranto \& Dantas-Torres, 2013). Antes de 2003, data da primeira impressão do manual de vigilância e controle da $\mathrm{LV}$, o combate às leishmanioses era realizado pelas diretrizes no decreto $\mathrm{N}^{\circ} 51.838$ de 1963 . Este decreto 
determinava a eliminação de reservatórios como medida de controle da LV e utilizava inquéritos epidemiológicos extensivos para descoberta e posterior eutanásia de cães infectados, bem como a investigação de outros animais reservatórios. Em áreas endêmicas, essas medidas de controle têm como justificativa a detecção de que casos caninos precedem casos humanos, sendo a prevalência de LVC maior que a de LV (Freitas et al., 2013; Nunes et al., 2010; Palatnik-de-Sousa et al., 2009). A eutanásia mostra-se um procedimento com baixa eficiência para controle da LV (Costa, 2011; Dietze et al., 1997) e alguns fatores são descritos, dentre eles o não monitoramento de outros mamíferos reservatórios urbanos (Dantas-Torres et al., 2018a) e as dificuldades da detecção e acompanhamento do animal suspeito/infectado (Werneck, 2014), apesar de diversas ferramentas serem utilizadas para o diagnóstico (Duthie et al., 2018; Solano-Gallego et al., 2017).

Considerar exclusivamente cães como responsáveis pela manutenção da endemia de LV minimiza as possibilidades de sucesso no controle epidemiológico, já que outros mamíferos podem dividir o papel de reservatórios, incluindo o ser humano, sendo comprovada a capacidade de Homo sapiens servir de reservatório para LV (Ferreira et al., 2018). Vale ressaltar que a capacidade de reservatório é dinâmica, indo além de pertencer a uma determinada espécie (Courtenay et al., 2017).

\section{Cães como vítima da Leishmaniose Visceral}

Com a eutanásia se tornando um conceito socialmente inaceitável (Zuben \& Donalísio, 2016), além de não demonstrar eficiência em reduzir o número de incidência e letalidade de LV (Dantas-Torres et al., 2018a; Sousa-Paula et al., 2019), surge uma dicotomia colocando o cão como vítima do parasitismo. No entanto, essa premissa não anula o fato deles permanecerem como reservatórios. Atualmente o cão é uma espécie que compõe a família brasileira, havendo mais de 52,2 milhões nos lares (IBGE, 2013), com uma tendência a aumentar. O relacionamento positivo com os cães pode trazer benefícios ao ser humano, com relatos na melhora da saúde cardiovascular, na redução do estresse pelo aumento na produção do hormônio ocitocina e redução do cortisol, na promoção do desenvolvimento emocional e cognitivo em crianças (Machado et al., 2008; O’Haire, 2010).

$\mathrm{O}$ aumento na proximidade com o ser humano, em decorrência de mudanças na estrutura familiar, promoveu uma maior formação de vínculo entre ambos, sendo hoje o cão considerado um membro da família (Rebelo, 2016). Essa relação tem se tornado tão estreita que o conceito de família multiespécie, formada por seres humanos e animais que têm um vínculo afetivo, tem se tornado popular e ganhado espaço no meio jurídico (Ś́guin et al., 2017). Este novo tipo de relacionamento torna a eutanásia mais difícil e dolorosa de serem realizadas (Esch et al., 2012; Zuben \& Donalísio, 2016).

Do ponto de vista clínico-veterinário, a eutanásia não é uma recomendação para todos os cães soropositivos, pois o diagnóstico e tratamento da LVC permite o estadiamento em até cinco estágios, sendo a eutanásia indicada nos últimos estágios (IV e V), de prognóstico reservado, ou quando não há compromisso da família em tratar o animal (Meléndez-Lazo et al., 2018; Passantino et al., 2010). Nos outros estágios, o animal pode viver anos sem muitas intervenções médicas (Abbehusen et al., 2017), mas deve ser acompanhado pelo médico veterinário, sendo adotadas medidas sanitárias para evitar propagação do parasito (Travi et al., 2018). Tais medidas consistem em evitar o contato do vetor com o cão em tratamento, diminuir a carga parasitária e impedir a transmissão sexual/vertical. Para evitar o contato do vetor, existem coleiras impregnadas com inseticidas (David et al., 2001), imersão em solução inseticida (Courtenay et al., 2009) e produtos spot on (Reithinger et al., 2001), que podem ser prescritos pelo médico veterinário. $\mathrm{O}$ uso de telas na casa e nos canis, bem como inseticidas é indicado. A diminuição da carga parasitária pode ser alcançada pelo tratamento clínico (Nogueira et al., 2019) e a reprodução pode ser impedida facilmente pelo isolamento do cão (de outros cães) ou castração.

Para os cães suscetíveis à infecção, existem várias ferramentas para prevenir LVC. No entanto, nenhuma ainda é 100\% eficaz (Miró et al., 2017; Papadopoulos et al., 2017). As medidas de prevenção sugeridas de LVC são utilização de repelentes de longa ação nos cães, seja na forma de coleiras impregnadas ou de produtos spot on, uso de vacinas quando disponíveis e manter o ambiente limpo de material orgânico e sujidades que possam atrair os flebotomíneos (Miró et al., 2017).Logo, o direito de tratar os cães infectados deve ser respeitado, mas o papel de reservatório de uma zoonose possivelmente fatal não deve ser negligenciado. Sugere-se como alternativa utilizar essa espécie de convívio próximo ao homem como sentinela. 


\section{Cão como sentinela da Leishmaniose Visceral}

O animal sentinela tem como finalidade detectar a exposição a determinados agentes ou contaminantes presentes no ambiente, identificando ameaças em potencial para seres humanos ou animais o que implica em preservar vidas (National Research Council, 1991). Bons sentinelas são aqueles que mimetizam a doença em humanos, são fáceis de manusear, são abundantes, possuem técnicas de diagnóstico da enfermidade padronizada e com desempenho semelhante ou superior a humanos (National Research Council, 1991). Ressalte-se que essas são características dos cães na LV. Eles são excelentes modelos para estudos de imunopatologia (Schaut et al., 2016; Toepp et al., 2018; Vida et al., 2016) para desenvolvimento de doença (Abbehusen et al., 2017) são abundantes de convívio próximo ao homem lares (IBGE, 2013) e apresentam maior suscetibilidade a infecção por L. infantum (Tabela 1). Isso torna o cão ideal para ser utilizado como espécie sentinela da LV.

\section{Uma nova abordagem dos cães na Leishmaniose Visceral}

A promoção de saúde que integre seres humanos, animais e meio ambiente, a Saúde Única, tem sido a meta de organizações como "World Organizaation for Animal Health" (OIE), "Food and Agriculture Organization of the United Nations" (FAO) e "World Health Organization" (WHO), na qual se busca incentivar e promover pesquisas para uma melhor compreensão das zoonoses. Neste sentido, é importante a sugestão de medidas que estimulem a saúde única.

No âmbito do PCV-LV deve-se incluir o monitoramento ativo de grupos de animais e humanos mais suscetíveis à infecção por $L$. infantum e encaminhá-los para as devidas medidas terapêutica-sanitária, o que implicaria na redução da carga parasitaria no ambiente. Neste monitoramento, sugere-se a inclusão de cães, gambás de orelha branca (Sherlock et al., 1984), raposas (Lainson et al., 1969), crianças até 12 anos (Mohebali et al., 2010) e adultos infectados com HIV (Ferreira et al., 2018).

Instituir um novo programa em um conceito de "one health" com o cão como animal sentinela deve ser prioridade. Considerar todas as espécies parte de um só ambiente onde humanos se beneficiam da saúde deste ambiente. Logo, o programa deveria ser sustentado por três pilares (Figura 3): 1. Redução da carga parasitaria do ambiente; 2. Redução da transmissibilidade no ecossistema; 3. Controle dos animais doentes.

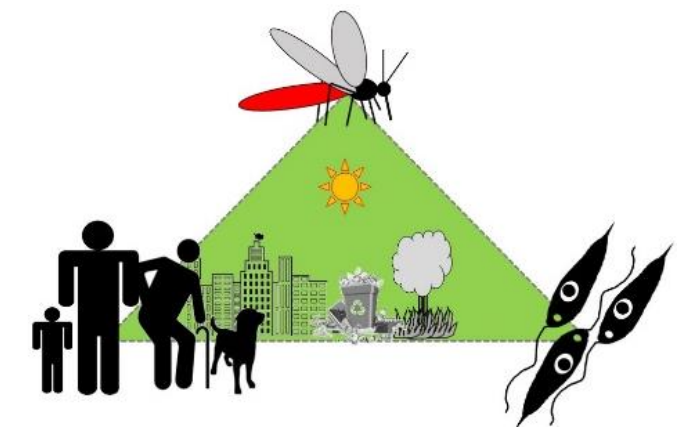

Figura 3. Tríade epidemiológica das Leishmanioses Visceral Humana e Canina. Os hospedeiros mamíferos, o vetor biológico flebotomíneo e o parasito $L$. infantum estão sujeitas as condições individuais e ambientais, que influenciam na transmissão da doença entre os elementos.

A redução da carga de parasitos circulantes no ambiente consiste em tratar ou eliminar animais e vetores contaminados. Essa medida tem um gargalo: dependência do método de diagnóstico para detectar os hospedeiros acometidos antes de disseminar o parasito. Atualmente, ainda existe uma demora no diagnóstico de casos humanos (Akhoundi et al., 2017) bem como de casos caninos (Teixeira et al., 2019). Existem dados de que cães soronegativos no Dual Path Plataform ${ }^{\circledR}$ (DPP) para LVC são competentes para infectar Lu. Longipalpis (Borja et al., 2016), enquanto que cães tratados para LVC parecem diminuir ou extinguir a capacidade de transmitir o parasito aos vetores testados (Nery et al., 2017; Nogueira et al., 2019). Estes fatos corroboram com a ideia de que o cão é hospedeiro e vítima do programa de controle vigente, e que o tratamento dos cães junto com a conscientização da população pode ser mais eficiente que as medidas atuais recomendadas. 
Diminuir a transmissibilidade no ambiente consiste em diminuir o número de vetores ou a exposição de animais contaminados. Espécies do gênero Leishmania possuem obrigatoriamente uma fase da vida no hospedeiro mamífero e outra fase no vetor biológico (Rougeron et al., 2017). No Brasil, os vetores biológicos são insetos fêmeas do gênero Lutzomyia, sendo a mais abundante a Lu. Longipalpis (Silva et al., 2018). A transmissão pelo vetor biológico é natural do parasito e forma a tríade hospedeiro-vetorparasito (Figura 3), já que o parasito depende do vetor para completar suas fases de desenvolvimento e maturação (Figura 2) (Sunter \& Gull, 2017). Considerando a transmissão enzoótica nos cães, uma importante medida é a castração de animais infectados.

O controle do vetor pela aplicação de inseticidas ambientais tem limitações devido ao pouco conhecimento dos hábitos do vetor biológico. Compara-se erroneamente o comportamento dos flebotomíneos com os culicídeos transmissores das arboviroses, consequentemente são utilizados métodos de borrifar inapropriados (Werneck et al., 2014). Logo, aspectos ecoepidemiológicos devem ser investigados a fim de se estabelecer medidas que possam reduzir a carga vetorial ambiental (Lana et al., 2018). Assim, o manejo ambiental com a remoção do excesso de matéria orgânica, a poda de árvores para exposição do solo à luz solar, o saneamento básico e o recolhimento do lixo, devem ser medidas implementadas para prevenir LVC (Lara-Silva et al., 2017).

A redução dos animais doentes vai além do tratamento. Inicialmente, deve-se realizar o diagnóstico de forma ativa nas populações mais suscetíveis para detectar aqueles animais sem manifestações clínicas da doença, mas disseminadores do parasito, e de forma passiva em hospedeiros suspeitos. A partir do diagnóstico preciso, inicia-se a terapêutica indicada para a espécie e a vigilância ativa na área do caso através da investigação de espécies suscetíveis, do manejo ambiental e do uso de técnicas que diminuam efetivamente a carga vetorial.

Estudos mostram que casos de LVC precedem casos humanos, tanto em novos surtos/epidemias (Mahmud et al., 2019) como em aumento/diminuição de incidência (Freitas et al., 2013; Lima et al., 2017; Costa et al., 2018). Quando o tratamento dos cães é realizado por tutores responsáveis e divulgado para população de forma adequada, juntamente com as medidas de prevenção contra o vetor e os cuidados ambientais, promove-se uma medida educativa no combate às Leishmanioses.

Prevenir a LVC torna-se importante pelo valor afetivo dos cães para as famílias (Séguin \& Descoteaux, 2016), por serem vítimas e reservatórios em potencial nos ambientes urbanos (Alencar, 1959; Prado et al., 2011). É necessário que o cão deixe de ser considerado o vilão da LV por ser um dos hospedeiros desta doença, e que a eliminação dos animais soropositivos não seja mais uma realidade (Dantas-Torres et al., 2019). Visando a prevenção de LV, torna-se imperativo o manejo ético e tratamento dos cães. Ressalta-se que o tratamento dos cães por tutores éticos e responsáveis precisa ser estimulado e divulgado nos meios de comunicação para que a sociedade se conscientize dos preceitos básicos de uma saúde única.

\section{Considerações finais}

A LVC permanece como um problema que aflige a população Brasileira, afetando cães que hoje são considerados membros da família. Pode-se observar que os métodos de controle e vigilância paras as leishmanioses viscerais implementadas no Brasil ainda são ineficientes e desatualizadas. Portanto, novos conhecimentos e medidas devem ser utilizados na adoção de programas que incluam a saúde única na forma de lidar com as leishmanioses: hospedeiros, vetores e parasitos dentro de um mesmo ambiente para dirigir recursos em medidas eficientes, usando o cão como sentinela da LV. Neste sentido, é preciso estimular a união de profissionais médicos, médicos veterinários, parasitologistas, sanitaristas e as famílias para que sejam aliados na implementação de medidas éticas e responsáveis para obtenção de resultados satisfatórios no controle da LV-LVC.

\section{Referências bibliográficas}

Abbehusen, M. M. C., Almeida, V. A., Solcà, M. da S., Pereira, L. S., Costa, D. J., Gil-Santana, L., Bozza, P. T., Fraga, D. B. M., Veras, P. S. T., \& Santos, W. L. C. (2017). Clinical and immunopathological findings during long term follow-up in Leishmania infantum experimentally infected dogs. Scientific Reports, 7(1), 1-11. https://doi.org/10.1038/s41598-017-15651-8. 
Abrantes, T. R., Werneck, G. L., Almeida, A. S., \& Figueiredo, F. B. (2018). Fatores ambientais associados à ocorrência de leishmaniose visceral canina em uma área de recente introdução da doença no Estado do Rio de Janeiro, Brasil. Cadernos de Saúde Pública, 34, 1-12.

Afonso, M. M., Gomes, A. C., Meneses, C. R. V., \& Rangel, E. F. (2005). Studies on the feeding habits of Lutzomyia (N.) intermedia (Diptera, Psychodidae), vector of cutaneous leishmaniasis in Brazil. Cadernos de Saúde Pública, 21, 1816-1820. https://doi.org/10.1590/S0102-311X2005000600030.

Akhoundi, M., Downing, T., Votýpka, J., Kuhls, K., Lukeš, J., Cannet, A., Ravel, C., Marty, P., Delaunay, P., \& Kasbari, M. (2017). Leishmania infections: Molecular targets and diagnosis. Molecular Aspects of Medicine, 57, 1-29. https://doi.org/10.1016/j.mam.2016.11.012.

Akhoundi, M., Kuhls, K., Cannet, A., Votýpka, J., Marty, P., Delaunay, P., \& Sereno, D. (2016). A historical overview of the classification, evolution, and dispersion of Leishmania parasites and sandflies. PLoS Neglected Tropical Diseases, 10(3), e0004349.

Alencar, J. E. (1959). Calazar canino. Contribuição para o estudo da epidemiologia do calazar no Brasil. Revista de La Facultad de Ciencias Médicas, 10(1-4), 177-179.

Almeida, A. B. P., Mendonça, A. J., \& Sousa, V. R. F. (2010). Prevalência e epidemiologia da leishmaniose visceral em cães e humanos, na cidade de Cuiabá, Mato Grosso, Brasil. Ciência Rural, 40(7), 1610-1615.

Andrade, H. M., Toledo, V., Pinheiro, M. B., Guimarães, T., Oliveira, N. C., Castro, J. A., Silva, R. N., Amorim, A. C., Brandão, R., \& Yoko, M. (2011). Evaluation of miltefosine for the treatment of dogs naturally infected with L. infantum (= L. chagasi) in Brazil. Veterinary Parasitology, 181(2-4), 8390. https://doi.org/10.1016/j.vetpar.2011.05.009.

Bekhit, A. A., El-Agroudy, E., Helmy, A., Ibrahim, T. M., Shavandi, A., \& Bekhit, A. E.-D. A. (2018). Leishmania treatment and prevention: Natural and synthesized drugs. European Journal of Medicinal Chemistry, 160, 229-244. https://doi.org/10.1016/j.ejmech.2018.10.022.

Belo, V. S., Gregório, E. A., Teixeira-Neto, R. G., da Rocha, A. C. V. M., Pereira, A. A. S., Marcelino, A. P., Paz, G. F., \& Silva, E. S. (2017). Reliability of techniques used in the diagnosis of canine visceral leishmaniasis by the national control program in Brazil: A survey in an area of recent transmission. Preventive Veterinary Medicine, 146, 10-15. https://doi.org/10.1016/j.prevetmed.2017.07.011.

Boggiatto, P. M., Gibson-Corley, K. N., Metz, K., Gallup, J. M., Hostetter, J. M., Mullin, K., \& Petersen, C. A. (2011). Transplacental transmission of Leishmania infantum as a means for continued disease incidence in North America. PLoS Neglected Tropical Diseases, 5(4), e1019. https://doi.org/10.1371/journal.pntd.0001019.

Borja, L. S., Sousa, O. M. F., Silva Solcà, M., Bastos, L. A., Bordoni, M., Magalhães, J. T., Larangeira, D. F., Barrouin-Melo, S. M., Fraga, D. B. M., \& Veras, P. S. T. (2016). Parasite load in the blood and skin of dogs naturally infected by Leishmania infantum is correlated with their capacity to infect sand fly vectors. Veterinary Parasitology, 229, 110-117. https://doi.org/10.1016/j.vetpar.2016.10.004.

Brasil.(2014). Manual de Vigilância e Controle da Leishmaniose Visceral. Disponível em: < http://bvsms.saude.gov.br/bvs/publicacoes/manual_vigilancia_controle_leishmaniose_visceral.pdf> . Acesso em: 25 jan., 2020

Brasil. (2016). Ministério da Saúde. Secretaria de Vigilância em Saúde. Coordenação-Geral de Desenvolvimento da Epidemiologia em Serviços. Guia de Vigilância em Saúde. Disponível em: < https://portalarquivos2.saude.gov.br/images/pdf/2016/novembro/18/Guia-LV-2016.pdf<. Acesso em: 12 abril, 2020.

Brito, F. G., Langoni, H., Silva, R. C., Rotondano, T. E. F., Melo, M. A., \& Paz, G. S. (2016). Canine visceral leishmaniasis in the Northeast Region of Brazil. Journal of Venomous Animals and Toxins Including Tropical Diseases, 22(1), 10-13. https://doi.org/10.1186/s40409-016-0069-4.

Carvalho, A. G., Luz, J. G. G., Rodrigues, L. D., Dias, J. V. L., \& Fontes, C. J. F. (2018). High seroprevalence and peripheral spatial distribution of visceral leishmaniasis among domestic dogs in an emerging urban focus in Central Brazil: a cross-sectional study. Pathogens and Global Health, 112(1), 29-36. https://doi.org/10.1080/20477724.2018.1438229. 
Costa, C. H. N. (2011). How effective is dog culling in controlling zoonotic visceral leishmaniasis? A critical evaluation of the science, politics and ethics behind this public health policy. Revista Da Sociedade Brasileira de Medicina Tropical, 44(2), 232-242. https://doi.org/10.1590/s003786822011005000014.

Costa, H. X. (2011). Interação de hemoparasitos e hemoparasitoses em casos clínicos de trombocitopenia em cães no município de Goiânia. In Ciência Animal: Vol. Masster of. Universidade Federal do Goiás.

Costa, D.N.C.C., Bermudi, P.M.M., Rodas, L.A.C., Nunes, C.M., Hiramoto, R.M., Tolezano, J. E., Cipriano, R. S., Cardoso, G. C. D., Codeço, C. T. \& Chiaravalloti-Neto, F. (2018). Human visceral leishmaniasis and relationship with vector and canine control measures. Revista de Saúde Pública, 52, 1-11. https://doi.org/10.11606/S1518-8787.2018052000381

Coura-Vital, W., Ker, H. G., Roatt, B. M., Aguiar-Soares, R. D. O., Leal, G. G. A., Moreira, N. D., Oliveira, L. A. M., Machado, E. M. M., Morais, M. H. F., \& Correa-Oliveira, R. (2014). Evaluation of change in canine diagnosis protocol adopted by the visceral leishmaniasis control program in Brazil and a new proposal for diagnosis. PLoS One, 9(3), e91009. https://doi.org/10.1371/journal.pone.0091009.

Courtenay, O, Kovacic, V., Gomes, P. A. F., Garcez, L. M., \& Quinnell, R. J. (2009). A long-lasting topical deltamethrin treatment to protect dogs against visceral leishmaniasis. Medical and Veterinary Entomology, 23(3), 245-256. https://doi.org/10.1111/j.1365-2915.2009.00815.x.

Courtenay, Orin, Peters, N. C., Rogers, M. E., \& Bern, C. (2017). Combining epidemiology with basic biology of sand flies, parasites, and hosts to inform leishmaniasis transmission dynamics and control. PLoS Pathogens, 13(10), e1006571. https://doi.org/10.1371/journal.ppat.1006571.

Courtenay, Orin, Quinnell, R. J., Garcez, L. M., Shaw, J. J., \& Dye, C. (2002). Infectiousness in a cohort of Brazilian dogs: why culling fails to control visceral leishmaniasis in areas of high transmission. The Journal of Infectious Diseases, 186(9), 1314-1320. https://doi.org/10.1086/344312.

Dantas-Torres, F. (2006). Leishmania infantum versus Leishmania chagasi: do not forget the law of priority. Memórias Do Instituto Oswaldo Cruz, 101(1), 117-118.

Dantas-Torres, F., Miró, G., Baneth, G., Bourdeau, P., Breitschwerdt, E., Capelli, G., Cardoso, L., Day, M. J., Dobler, G., \& Ferrer, L. (2019). Canine leishmaniasis control in the context of One Health. Emerging Infectious Diseases, 25(12), 1-4. https://doi.org/10.3201/eid2512.190164.

Dantas-Torres, F., Miró, G., Bowman, D. D., Gradoni, L., \& Otranto, D. (2018a). Culling dogs for zoonotic visceral leishmaniasis control: the wind of change. Trends in Parasitology, 35(2), 97-101. https://doi.org/10.1016/j.pt.2018.11.005.

Dantas-Torres, F., Sales, K.G.S., da Silva, L.G., Otranto, D. \& Figueredo, L.A (2018b). Level of agreement between two commercially available rapid serological tests and the official screening test used to detect Leishmania seropositive dogs in Brazil. The Veterinary Journal, 234, 102-104. https://doi.org/10.1016/j.tvj1.2018.02.007.

David, J. R., Stamm, L. M., Bezerra, H. S., Souza, R. N., Killick-Kendrick, R., \& Lima, J. W. O. (2001). Deltamethrin-impregnated dog collars have a potent anti-feeding and insecticidal effect on Lutzomyia longipalpis and Lutzomyia migonei. Memórias Do Instituto Oswaldo Cruz, 96, 839-847. https://doi.org/10.1590/S0074-02762001000600018.

Dietze, R., Barros, G. B., Teixeira, L., Harris, J., Michelson, K., Falqueto, A., \& Corey, R. (1997). Effect of eliminating seropositive canines on the transmission of visceral leishmaniasis in Brazil. Clinical Infectious Diseases, 25(5), 1240-1242. https://doi.org/10.1086/516096.

Duthie, M. S., Lison, A., \& Courtenay, O. (2018). Advances toward diagnostic tools for managing zoonotic visceral leishmaniasis. Trends in Parasitology, 34(10), 881-890. https://doi.org/10.1016/j.pt.2018.07.012.

Esch, K. J., Pontes, N. N., Arruda, P., O’Connor, A., Morais, L., Jeronimo, S. M. B., \& Petersen, C. A. (2012). Preventing zoonotic canine leishmaniasis in northeastern Brazil: pet attachment and adoption of community leishmania prevention. The American Journal of Tropical Medicine and Hygiene, 87(5), 822-831. https://doi.org/10.4269/ajtmh.2012.12-0251. 
Evans, B. R., \& Leighton, F. A. (2014). A history of One Health. Revue Scientifique et Technique, 33(2), 413-420.

Ferreira, G. R., Ribeiro, J. C. C. B., Meneses Filho, A., Pereira, T. J. C. F., Parente, D. M., Pereira, H. F., Silva, J. C., Zacarias, D. A., Silva, L. V., \& Faustino, S. K. M. (2018). Human competence to transmit Leishmania infantum to Lutzomyia longipalpis and the influence of human immunodeficiency virus infection. The American Journal of Tropical Medicine and Hygiene, 98(1), 126. https://doi.org/10.4269/ajtmh.16-0883.

Figueiredo, F. B., Vasconcelos, T. C. B., Madeira, M. de F., Menezes, R. C., Maia-Elkhoury, A. N. S., Marcelino, A. P., \& Werneck, G. L. (2018). Validation of the Dual-path Platform chromatographic immunoassay (DPP ${ }^{\circ}$ CVL rapid test) for the serodiagnosis of canine visceral leishmaniasis. Memórias Do Instituto Oswaldo Cruz, 113(11), 1-7. https://doi.org/10.1590/0074-02760180260.

Fraga, Deborah B M, Solcà, M. S., Silva, V. M. G., Borja, L. S., Nascimento, E. G., Oliveira, G. G. S., Pontes-de-Carvalho, L. C., Veras, P. S. T., \& Santos, W. L. C. (2012). Temporal distribution of positive results of tests for detecting Leishmania infection in stray dogs of an endemic area of visceral leishmaniasis in the Brazilian tropics: A 13 years survey and association with human disease. Veterinary Parasitology, 190(3-4), 591-594.

Fraga, Deborah Bittencourt Mothé, Pacheco, L. V., Borja, L. S., Tuy, P. G. da S. E., Bastos, L. A., Solcà, M. da S., Amorim, L. D. A. F., \& Veras, P. S. T. (2016). The rapid test based on Leishmania infantum chimeric rK28 protein improves the diagnosis of canine visceral leishmaniasis by reducing the detection of false-positive dogs. PLoS Neglected Tropical Diseases, 10(1), e0004333. https://doi.org/10.1371/journal.pntd.0004333.

Freitas, J. C. C., Sampaio Filho, A. P., Santos, G. J. L., Lima, A. L., \& Nunes-Pinheiro, D. C. S. (2013). Analysis of seasonality, tendencies and correlations in human and canine visceral leishmaniasis. Acta Scientiae Veterinariae, 41(1), 1-8.

Gonçalves, M. B. (2014). Prevalência, distribuição e identificação de prováveis fatores de risco para Leishmaniose Visceral canina em Camaçari - BA. 113. Fundação Oswaldo Cruz, Centro de Pesquisas Gonçalo Moniz, Salvador, 113f.

Góes, M. A. O., Melo, C. M., \& Jeraldo, V. D. L. S. (2012). Série temporal da leishmaniose visceral em Aracaju, estado de Sergipe, Brasil (1999 a 2008): aspectos humanos e caninos. Revista Brasileira de Epidemiologia, 15(2), 298-307.

IBGE. (2013). População de Animais de Estimação no Brasil - 2013 - n.d. Disponível em: http://www.agricultura.gov.br/assuntos/camaras-setoriais-tematicas/documentos/camarastematicas/insumos-agropecuarios/anos-anteriores/ibge-populacao-de-animais-de-estimacao-nobrasil-2013-abinpet-79.pdf/view. Acesso em: 12 nov. 2019.

Irwin, P. J. (2014). It shouldn't happen to a dog or a veterinarian: clinical paradigms for canine vectorborne diseases. Trends in Parasitology, 30(2), 104-112. https://doi.org/10.1016/j.pt.2013.12.001.

Kazimoto, T. A., Amora, S. S. A., Figueiredo, F. B., Magalhães, J. M., Freitas, Y. B. N., Sousa, M. L. R., Melo, A. E. C. da S., Campos, M. P., Alves, N. D., \& Werneck, G. L. (2018). Impact of 4\% deltamethrin-impregnated dog collars on the prevalence and incidence of canine visceral leishmaniasis. Vector-Borne and Zoonotic Diseases, 18(7), 356-363. https://doi.org/10.1089/vbz.2017.2166.

Lainson, R., Shaw, J. J., \& Lins, Z. C. (1969). Leishmaniasis in Brazil IV: the fox, Cerdocyon thous (L) as a reservoir of Leishmania donovani in Para State, Brazil. 63, 741-745. https://doi.org/10.1016/0035-9203(69)90118-7.

Lana, R. S., Michalsky, É. M., Lopes, L. O., Lara-Silva, F. O., Nascimento, J. L., Pinheiro, L. C., FrançaSilva, J. C., Mendes, T. S. C., Fortes-Dias, C. L., \& Dias, E. S. (2018). Ecoepidemiological aspects of visceral leishmaniasis in an endemic area in the Steel Valley in Brazil: an ecological approach with spatial analysis. PLoS One, 13(10), e0206452. https://doi.org/10.1371/journal.pone.0206452.

Lara-Silva, F. O., Michalsky, É. M., Fortes-Dias, C. L., Fiuza, V. O. P., \& Dias, E. S. (2017). Evaluation of chemical spraying and environmental management efficacy in areas with minor previous application of integrated control actions for visceral leishmaniasis in Brazil. Acta Tropica, 176, 109113. https://doi.org/10.1016/j.actatropica.2017.07.029. 
Leal, G. G. A., Carneiro, M., Pinheiro, A. C., Marques, L. A., Ker, H. G., Reis, A. B., \& Coura-Vital, W. (2018). Risk profile for Leishmania infection in dogs coming from an area of visceral leishmaniasis reemergence. Preventive Veterinary Medicine, 150, 1-7. https://doi.org/10.1016/j.prevetmed.2017.11.022.

Leça Júnior, N. F., Guedes, P. E. B., Santana, L. N., Anjos Almeida, V., Carvalho, F. S., Albuquerque, G. R., Wenceslau, A. A., Munhoz, A. D., \& Silva, F. L. (2015). Epidemiology of canine leishmaniasis in southern Bahia, Brazil. Acta Tropica, 148, 115-119.

Lima, Á. L. M., Lima, I. D., Coutinho, J. F. V, Sousa, Ú. P. S. T., Rodrigues, M. A. G., Wilson, M. E., Pearson, R. D., Queiroz, J. W., \& Jerônimo, S. M. B. (2017). Changing epidemiology of visceral leishmaniasis in northeastern Brazil: a 25-year follow-up of an urban outbreak. Transactions of the Royal Society of Tropical Medicine and Hygiene, 111(10), 440-447.

Lima, I. D., Lima, A. L. M., Mendes-Aguiar, C. de O., Coutinho, J. F. V, Wilson, M. E., Pearson, R. D., Queiroz, J. W., \& Jeronimo, S. M. B. (2018). Changing demographics of visceral leishmaniasis in northeast Brazil: Lessons for the future. PLoS Neglected Tropical Diseases, 12(3), e0006164.

Machado, J. D. A. C., Rocha, J. R., Santos, L. M., \& Piccinin, A. (2008). Terapia assistida por animais (TAA). Revista Científica Eletrônica de Medicina Veterinária, 10, 1-7.

Mahmud, I. C., Piassini, L. de A. S., Motta, F., Behar, P. R. P., \& Souza, G. D. (2019). Epidemiological aspects of the first human autochthonous visceral leishmaniosis cases in Porto Alegre, Brazil. Brazilian Journal of Infectious Diseases, 23(2), 124-129. https://doi.org/10.1016/j.bjid.2019.04.004.

Manna, L., Reale, S., Vitale, F., Picillo, E., Pavone, L. M., \& Gravino, A. E. (2008). Real-time PCR assay in Leishmania-infected dogs treated with meglumine antimoniate and allopurinol. The Veterinary Journal, 177(2), 279-282. https://doi.org/10.1016/j.tvj1.2007.04.013.

Meléndez-Lazo, A., Ordeix, L., Planellas, M., Pastor, J., \& Solano-Gallego, L. (2018). Clinicopathological findings in sick dogs naturally infected with Leishmania infantum: Comparison of five different clinical classification systems. Research in Veterinary Science, 117, 18-27. https://doi.org/10.1016/j.rvsc.2017.10.011.

Miró, G., Petersen, C., Cardoso, L., Bourdeau, P., Baneth, G., Solano-Gallego, L., Pennisi, M. G., Ferrer, L., \& Oliva, G. (2017). Novel areas for prevention and control of canine leishmaniosis. Trends in Parasitology, 33(9), 718-730. https://doi.org/10.1016/j.pt.2017.05.005.

Mohebali, M., Edrissian, G. H., Shirzadi, M. R., Hosseingholizadeh, G., Pashaei, M. H., Ganji, A., Zarei, Z., Kousha, A., Akhoundi, B., \& Hajjaran, H. (2010). Integrated visceral leishmaniasis surveillance system in primary care for children in Meshkin-Shahr district, north-western Islamic Republic of Iran. Eastern Mediterranean Health Journal, 16(10), 1050-1054. https://doi.org/10.26719/2010.16.10.1050.

National Research Council. (1991). Animals as Sentinels of Environmental Health Hazards. Washington, D.C.: National Academies Press.

Nery, G., Becerra, D. R. D., Borja, L. S., Souza, B. M. P. S., Franke, C. R., Veras, P. S. T., Larangeira, D. F., \& Barrouin-Melo, S. M. (2017). Avaliação da infectividade parasitária a Lutzomyia longipalpis por xenodiagnóstico em cães tratados para leishmaniose visceral naturalmente adquirida. Pesquisa Veterinária Brasileira, 37, 701-707. https://doi.org/10.1590/s0100-736x2017000700009.

Nogueira, F. S., Avino, V. C., Galvis-Ovallos, F., Pereira-Chioccola, V. L., Moreira, M. A. B., Romariz, A. P. P. L., Molla, L. M., \& Menz, I. (2019). Use of miltefosine to treat canine visceral leishmaniasis caused by Leishmania infantum in Brazil. Parasites \& Vectors, 12(1), 1-11. https://doi.org/10.1186/s13071-019-3323-0.

Nunes, C. M., Pires, M. M., Silva, K. M., Assis, F. D., Gonçalves Filho, J., \& Perri, S. H. V. (2010). Relationship between dog culling and incidence of human visceral leishmaniasis in an endemic area. Veterinary Parasitology, 170(1-2), 131-133. https://doi.org/10.1016/j.vetpar.2010.01.044.

O'Haire, M. (2010). Companion animals and human health: Benefits, challenges, and the road ahead. Journal of Veterinary Behavior, 5(5), 226-234. https://doi.org/10.20506/rst.37.1.2741.

Oikonomidis, I. L., Tsouloufi, T. K., Mylonakis, M. E., Psalla, D., Soubasis, N., Rallis, T., \& KritsepiKonstantinou, M. (2019). Circulating and bone marrow myeloid cells containing Leishmania 
amastigotes in a case of advanced canine leishmaniosis. Journal of Veterinary Diagnostic Investigation, 31(5), 726-731.

Oliveira, A. R., \& Fernandes, C. A. (2014). Focos e fatores associados ao aparecimento de leishmaniose tegumentar americana (lta) e leishmaniose visceral (lv) no Cariri Cearense. Revista Interfaces: Saúde, Humanas e Tecnologia, 2(5).

Organização Pan-Americana Saúde (OPAS). (2018). Leishmanioses: Informe epidemiológico das américas. Disponível em: < https://iris.paho.org/bitstream/handle/10665.2/34857/LeishReport6_por.pdf?sequence=5\#: :text=A $\% 20 \operatorname{taxa} \% 20 \mathrm{de} \% 20 \mathrm{incid} \% \mathrm{C} 3 \%$ AAncia\%20foi, $\% 2 \mathrm{C} 35 \% 2 \mathrm{~F} 100.000 \% 20 \mathrm{habitantes})>$. Acesso em: 14 maio, 2020.

Otranto, D., \& Dantas-Torres, F. (2013). The prevention of canine leishmaniasis and its impact on public health. Trends in Parasitology, 29(7), 339-345. https://doi.org/10.1016/j.pt.2013.05.003.

Palatnik-de-Sousa, C. B., Silva-Antunes, I., de Aguiar Morgado, A., Menz, I., Palatnik, M., \& Lavor, C. (2009). Decrease of the incidence of human and canine visceral leishmaniasis after dog vaccination with Leishmune ${ }^{\circledR}$ in Brazilian endemic areas. Vaccine, 27(27), 3505-3512.

Papadopoulos, E., Angelou, A., Diakou, A., Halos, L., \& Beugnet, F. (2017). Five-month serological monitoring to assess the effectiveness of permethrin/fipronil (Frontline Tri-Act $\circledast$ ) spot-on in reducing the transmission of Leishmania infantum in dogs. Veterinary Parasitology: Regional Studies and Reports, 7, 48-53. https://doi.org/10.1016/j.vprsr.2016.12.005.

Passantino, A., Russo, M., \& Coluccio, P. (2010). Canine leishmaniosis and euthanasia in Italy: a critical legal-ethical analysis. Revue Scientifique et Technique, 29(3), 537. https://doi.org/10.20506/rst.29.3.1993.

Pennisi, M. G., \& Persichetti, M. F. (2018). Feline leishmaniosis: Is the cat a small dog? Veterinary Parasitology, 251, 131-137. https://doi.org/10.1016/j.vetpar.2018.01.012.

Prado, P. F., Rocha, M. F., Sousa, J. F., Caldeira, D. I., Paz, G. F., \& Dias, E. S. (2011). Epidemiological aspects of human and canine visceral leishmaniasis in Montes Claros, State of Minas Gerais, Brazil, between 2007 and 2009. Revista Da Sociedade Brasileira de Medicina Tropical, 44(5), 561-566. https://doi.org/10.1590/s0037-86822011000500006.

Rebelo, Z. I. L. (2016). Caracterização dos comportamentos de tutores de animais de estimação numa amostra não probabilística. Universidade Lusófona de Humanidades $\mathrm{r}$ Tecnologia.

Reithinger, R., Teodoro, U., \& Davies, C. R. (2001). Topical insecticide treatments to protect dogs from sand fly vectors of leishmaniasis. Emerging Infectious Diseases, 7(5), 872-876. https://doi.org/10.3201/eid0705.017516.

Rezende, M. B., Herrera, H. M., Carvalho, C. M. E., Anjos, E. A. C., Ramos, C. A. N., De Araújo, F. R., Torres, J. M., \& De Oliveira, C. E. (2017). Detection of Leishmania spp. in bats from an area of Brazil endemic for visceral leishmaniasis. Transboundary and Emerging Diseases, 64(6), e36-e42. https://doi.org/10.1111/tbed.12597.

Ribeiro, C. R., Gonçalves, C. A., Cruz, L. M., \& Galera, P. D. (2019). Prevalência da leishmaniose visceral canina e coinfecções em região periurbana no Distrito Federal-Brasil. Ciência Animal Brasileira, 20, 1-10.

Rocha, M. A. N., Matos-Rocha, T. J., Ribeiro, C. M. B., \& Abreu, S. R. O. (2018). Epidemiological aspects of human and canine visceral leishmaniasis in State of Alagoas, Northeast, Brazil. Brazilian Journal of Biology, 78, 609-614. https://doi.org/10.1590/1519-6984.166622.

Rodrigues, A. C. M., Melo, A. C. F. L., Júnior, A. D. S., Franco, S. O., Rondon, F., \& Bevilaqua, C. M. L. (2017). Epidemiologia da leishmaniose visceral no município de Fortaleza, Ceará. Pesquisa Veterinária Brasileira, 37, 1119-1124. https://doi.org/10.1590/S0100-736X2017001000013.

Rondon, F. C. M., Bevilaqua, C. M. L., Franke, C. R., Barros, R. S., Oliveira, F. R., Alcântara, A. C., \& Diniz, A. T. (2008). Cross-sectional serological study of canine Leishmania infection in Fortaleza, Ceará state, Brazil. Veterinary Parasitology, 155(1-2), 24-31. https://doi.org/10.1016/j.vetpar.2008.04.014.

Roque, A. L. R., \& Jansen, A. M. (2014). Wild and synanthropic reservoirs of Leishmania species in 
the Americas. International Journal for Parasitology: Parasites and Wildlife, 3(3), 251-262. https://doi.org/10.1016/j.ijppaw.2014.08.004.

Rougeron, V., De Meeûs, T., \& Bañuls, A.-L. (2017). Reproduction in Leishmania: a focus on genetic exchange. Infection, Genetics and Evolution, 50, 128-132. https://doi.org/10.1016/j.meegid.2016.10.013.

Santos, S. O., Arias, J., Rribeiro, A. A., Hoffmann, M. P., Freitas, R. A., \& Malacco, M. A. F. (1998). Incrimination of Lutzomyia cruzi as a vector of American visceral leishmaniasis. Medical and Veterinary Entomology, 12(3), 315-317.

Schaut, R. G., Grinnage-Pulley, T. L., Esch, K. J., Toepp, A. J., Duthie, M. S., Howard, R. F., Reed, S. G., \& Petersen, C. A. (2016). Recovery of antigen-specific T cell responses from dogs infected with Leishmania (L.) infantum by use of vaccine associated TLR-agonist adjuvant. Vaccine, 34(44), 5225-5234. https://doi.org/10.1016/j.vaccine.2016.09.016.

Séguin, É., Araújo, L. M., \& Cordeiro Neto, M. R. C. (2017). Uma nova família: a multiespécie. Revista de Direito, 2017, 1-30.

Séguin, O., \& Descoteaux, A. (2016). Leishmania, the phagosome, and host responses: The journey of a parasite. Cellular Imunology, 309, 1-6.

Sherlock, Í. A., Miranda, J. C., Sadigursky, M., \& Grimaldi Júnior, G. (1984). Natural infection of the opossum Didelphis albiventris (Marsupialia, Didelphidae) with Leishmania donovani, in Brazil. Memórias Do Instituto Oswaldo Cruz, 79(4), 511. https://doi.org/10.1590/S007402761988000100019.

Silva, F. L., Oliveira, R. G., Silva, T. M. A., Xavier, M. N., Nascimento, E. F., \& Santos, R. L. (2009). Venereal transmission of canine visceral leishmaniasis. Veterinary Parasitology, 160(1-2), 55-59. https://doi.org/10.1016/j.vetpar.2008.10.079.

Silva, R. A., Andrade, A. J., Quint, B. B., Raffoul, G. E. S., Werneck, G. L., Rangel, E. F., \& Romero, G. A. S. (2018). Effectiveness of dog collars impregnated with $4 \%$ deltamethrin in controlling visceral leishmaniasis in Lutzomyia longipalpis (Diptera: Psychodidade: Phlebotominae) populations. Memorias Do Instituto Oswaldo Cruz, 113(5), 1-9. https://doi.org/10.1590/007402760170377.

Silva, R., Mendes, R. S., Santana, V. L., Souza, H. C., Ramos, C. P. S., Souza, A. P., Andrade, P. P., \& Melo, M. A. (2016). Aspectos epidemiológicos da leishmaniose visceral canina na zona rural do semiárido paraibano e análise de técnicas de diagnóstico. Pesquisa Veterinária Brasileira, 36, 625629. https://doi.org/10.1590/S0100-736X2016000700011.

Solano-Gallego, L., Cardoso, L., Pennisi, M. G., Petersen, C., Bourdeau, P., Oliva, G., Miró, G., Ferrer, L., \& Baneth, G. (2017). Diagnostic challenges in the era of canine Leishmania infantum vaccines. Trends in Parasitology, 33(9), 706-717. https://doi.org/10.1016/j.pt.2017.06.004.

Sousa-Paula, L. C., Silva, L. G., Sales, K. G. S., \& Dantas-Torres, F. (2019). Failure of the dog culling strategy in controlling human visceral leishmaniasis in Brazil: A screening coverage issue? PLoS Neglected Tropical Diseases, 13(6), e0007553. https://doi.org/10.1371/journal.pntd.0007553.

Sunter, J., \& Gull, K. (2017). Shape, form, function and Leishmania pathogenicity: from textbook descriptions to biological understanding. Open Biology, 7(9), 170165. https://doi.org/10.1098/rsob.170165.

Teixeira, A. I. P., Silva, D. M., Vital, T., Nitz, N., Carvalho, B. C., Hecht, M., Oliveira, D., Oliveira, E., Rabello, A., \& Romero, G. A. S. (2019). Improving the reference standard for the diagnosis of canine visceral leishmaniasis: a challenge for current and future tests. Memórias Do Instituto Oswaldo Cruz, 114, 1-9. https://doi.org/10.1590/0074-02760180452.

Toepp, A. J., Bennett, C., Scott, B., Senesac, R., Oleson, J. J., \& Petersen, C. A. (2019). Maternal Leishmania infantum infection status has significant impact on leishmaniasis in offspring. PLoS Neglected Tropical Diseases, 13(2), e0007058. https://doi.org/10.1371/journal.pntd.0007058.

Toepp, A., Larson, M., Wilson, G., Grinnage-Pulley, T., Bennett, C., Leal-Lima, A., Anderson, B., Parrish, M., Anderson, M., \& Fowler, H. (2018). Randomized, controlled, double-blinded field trial 
to assess Leishmania vaccine effectiveness as immunotherapy for canine leishmaniosis. Vaccine, 36(43), 6433-6441. https://doi.org/10.1016/j.vaccine.2018.08.087.

Travi, B. L., Cordeiro-da-Silva, A., Dantas-Torres, F., \& Miró, G. (2018). Canine visceral leishmaniasis: Diagnosis and management of the reservoir living among us. PLoS Neglected Tropical Diseases, 12(1), e0006082. https://doi.org/10.1371/journal.pntd.0006082.

Ursine, R. L., Paranaíba, L. F., Dias, J. V. L., Morais, H. A., \& Pires, H. H. R. (2016). Aspectos epidemiológicos da Leishmaniose Visceral humana e canina em municípios pertencentes à Superintendência Regional de Saúde de Diamantina, Minas Gerais, Brasil (2007-2012). Tempus Actas de Saúde Coletiva, 10(1), 179-193.

Vida, B., Toepp, A., Schaut, R. G., Esch, K. J., Juelsgaard, R., Shimak, R. M., \& Petersen, C. A. (2016). Immunologic progression of canine leishmaniosis following vertical transmission in United States dogs. Veterinary Immunology and Immunopathology, 169, 34-38. https://doi.org/10.1016/j.vetimm.2015.11.008.

Werneck, Guilherme L, Costa, C. H. N., Carvalho, F. A. A., Pires e Cruz, M. do S., Maguire, J. H., \& Castro, M. C. (2014). Effectiveness of insecticide spraying and culling of dogs on the incidence of Leishmania infantum infection in humans: a cluster randomized trial in Teresina, Brazil. PLoS Neglected Tropical Diseases, 8(10), e3172. https://doi.org/10.1371/journal.pntd.0003172.

Werneck, Guilherme Loureiro. (2014). Visceral leishmaniasis in Brazil: rationale and concerns related to reservoir control. Revista de Saúde Pública, 48, 851-856. https://doi.org/10.1590/S00348910.2014048005615.

WHO. (2010). Control of the Leishmaniases. World Health Organ Technical Report Series. Disponível em:https://apps.who.int/iris/handle/10665/44412. Acesso em: 20 dez. 2019.

World Health Organization (WHO). (2017). Global leishmaniasis update, 2006-2015: a turning point in leishmaniasis surveillance. Weekly eídemiological record, 92, 557-565.

Woerly, V., Maynard, L., Sanquer, A., \& Eun, H.-M. (2009). Clinical efficacy and tolerance of miltefosine in the treatment of canine leishmaniosis. Parasitology Research, 105(2), 463-469. https://doi.org/10.1007/s00436-009-1404-2.

Yasur-Landau, D., Jaffe, C. L., David, L., \& Baneth, G. (2016). Allopurinol resistance in Leishmania infantum from dogs with disease relapse. PLoS Neglected Tropical Diseases, 10(1), e0004341. https://doi.org/10.1371/journal.pntd.0004341.

Zinsstag, J., Meisser, A., Schelling, E., Tanner, M., \& Bonfoh, B. (2012). From'two medicines' to'One Health'and beyond: proceeding. Onderstepoort Journal of Veterinary Research, 79(2), 1-5.

Zuben, A. P. B. von, \& Donalísio, M. R. (2016). Difficulties in implementing the guidelines of the Brazilian Visceral Leishmaniasis Control Program in large cities. Cadernos de Saude Pública, 32(6), 1-11. https://doi.org/10.1590/0102-311X00087415.

Histórico do artigo:

Recebido: 16 de junho de 2021

Aprovado: 20 de agosto de 2021
Licenciamento: Este artigo é publicado na modalidade Acesso Aberto sob a licença Creative Commons Atribuição 4.0 (CC-BY 4.0), a qual permite uso irrestrito, distribuição, reprodução em qualquer meio, desde que $\mathrm{o}$ autor e a fonte sejam devidamente creditados. 